\title{
Imagery Rescripting for Reducing Body Image Dissatisfaction: A Randomized Controlled Trial
}

\author{
Ata Ghaderi ${ }^{1,2}$ (E) Elisabeth Welch ${ }^{2,3} \cdot$ Cecilia Zha $^{1} \cdot$ Emily A. Holmes $^{1,4}$
}

Accepted: 6 January 2022 / Published online: 12 February 2022

(c) The Author(s) 2022

\begin{abstract}
Background Brief, innovative, mechanistically-driven psychological treatments for body dissatisfaction are needed. We aimed to explore the occurrence of body-related mental images among females reporting a subjective sense of body dissatisfaction (study 1), and to investigate the potential efficacy of a single session of imagery rescripting (ImRS) to reduce body dissatisfaction (Study 2: pilot; Study 3 randomized controlled trial).

Methods and Results In study $1(\mathrm{n}=31)$, participants reported occurrence of both positive and negative body-related mental imagery. Frequency of body-related mental imagery and negative valence of such images were significantly related to body dissatisfaction. The pilot trial $(n=63)$ indicated feasibility, whereby ImRS of a distressing body-related mental image significantly reduced the believability, vividness, negative affect and distress of the image within-session and one-week post-session versus an active placebo condition (Expressive writing about body related thoughts and emotions). However, ImRS did not result in significant changes in body dissatisfaction. Study 3 was a randomized controlled trial $(n=113)$ with 3 conditions-ImRS, Expressive writing, and wait-list control. ImRS resulted in a significant reduction of vividness, controllability and negative affect caused by the image compared to Expressive writing condition, after the intervention. However, there were no significant reductions in body dissatisfaction from baseline to 1-week assessment, nor in post-hoc analyses at the 1-month follow-up. Findings suggest that a single session of imagery rescripting seems to alleviate the negative properties of the image (i.e., has proximal effects as hypothesized in our mechanistic account), but does not bring about a significant change in body dissatisfaction (i.e., transfer downstream to more distal effects).

Conclusions Given some promising effects of ImRS on negative body-related emotions, but lack of a downstream effect on body dissatisfaction itself, further investigation of the format, dose and focus of ImRS in this context is warranted.
\end{abstract}

Keywords Body image $\cdot$ Body dissatisfaction $\cdot$ Mental imagery $\cdot$ Imagery rescripting $\cdot$ Prevention

\section{Introduction}

Ata Ghaderi

ata.ghaderi@ki.se

1 Department of Clinical Neuroscience, Division of Psychology, Karolinska Institutet, 17177 Stockholm, Sweden

2 Stockholm Health Care Services, Region Stockholm, Stockholm Center for Eating Disorders, Stockholm, Sweden

3 Department of Clinical Neuroscience, Centre for Psychiatry Research, Karolinska Institutet, Stockholm, Sweden

4 Department of Psychology, Uppsala University, Uppsala, Sweden
Body image dissatisfaction, or body dissatisfaction is a common problem in Western cultures (Fallon et al., 2014). A review of research on body dissatisfaction reported a prevalence rate of $11-71 \%$ among females and $8-61 \%$ among males (Fiske et al., 2014). The large variation in prevalence rates reflected methodological issues such as use of one-item versus psychometrically sound measures, and samples. A study, using operational definitions of body dissatisfaction from previous research found a prevalence rate of $13-32 \%$ among females, and 9-28\% among males (Fallon et al., 2014). Body dissatisfaction is associated with poorer quality of life in adults (Mond et al., 2013) as well as adolescents (Griffiths et al., 2017), and this persists even after controlling for symptom of eating disorders, and body mass index 
(Griffiths et al., 2017). It prospectively predicts depressive mood and low self-esteem (Paxton et al., 2006), and it is a well-known risk factor for future onset of eating disorders (Ghaderi, 2003; Stice et al., 2002). A meta-analysis of standalone interventions for body image concerns (Alleva et al., 2015), and a recent meta-analysis of a dissonance-based preventive interventions for eating disorders (Stice et al., 2019) have shown only small improvements in body image.

We have argued for the need for brief, mechanistically driven, and scalable interventions to improve mental health treatments (Holmes et al., 2018). Such an approach includes new intervention approaches for body dissatisfaction, given its high prevalence, its status as a risk factor for eating disorder, and modest efficiency of current interventions for body dissatisfaction. Alleva and colleagues (2015) identified several specific techniques that were associated with improvement in body image. Guided imagery was one of the strategies employed to target experiential and behavioral avoidance (Alleva et al., 2015), factors hypothesized to maintain negative body image. Although guided imagery has been proposed as a potent treatment for body dissatisfaction and eating disorders for many years (Kearney-Cooke \& Striegel-Moore, 1997), empirical evaluations of this approach are more recent (Pennesi \& Wade, 2018). Although some earlier evaluations also exist (Riva, 1998; Riva et al., 2003), they are adapted for using virtual reality and combined with other cognitive behavioral techniques. Mental imagery has also been recently evaluated for the treatment of body dysmorphic disorder with promising outcomes (Ritter \& Stangier, 2016), which also adds to justification for systematic evaluation of such techniques for body dissatisfaction. However, guided imagery can be done in different ways with various levels of complexity. For mechanistic evaluation of effect of mental imagery on body dissatisfaction, the procedure should be highly specified, and the mechanisms through which it might exert its effect on outcome require investigation.

Mental imagery occurs when perceptual information is brought to mind in the absence of the relevant external stimuli (Pearson et al., 2015). Images are characterized by their subjective resemblance to different sensory modalities and can thus be associated with components of visual, auditory, olfactory or haptic sensations (Holmes \& Mathews, 2010). This may provide a possible explanation to why emotions and core beliefs can be more powerfully evoked and influenced through imagery compared to common cognitive strategies (e.g., verbal cognitive restructuring). Experimental work (e.g., Holmes \& Mathews, 2010; Holmes et al., 2008) demonstrated that imagery has a greater impact on emotions than verbal processing of equivalent stimuli. By asking a person to imagine a troublesome memory-or other forms of unwanted image e.g., a future concern-in the present, and to then subsequently intervene to change its meaning in a more desired way (i.e., imagery rescripting: ImRS), the negative meanings linked to the image can change. There is now growing interest in the use and evaluation of mental imagery as an adjunct or stand-alone treatment for several conditions such as PTSD or depression (Arntz, 2011; Wheatley et al., 2007) and mental imagery is recognized as a transdiagnostic process involved in a range of psychopathologies (Cili \& Stopa, 2015; Morina et al., 2017; Romano et al., 2020). "Based on the dual coding approach to working memory (Baddeley \& Andrade, 2000; Baddeley \& Hitch, 1974), any representation brought to mind of body image would most probably contain a visuospatial component alongside a verbal one, as also noted by other researchers (Darling et al., 2015). That is, considering one's own body image should, at least to some extent, invoke a visual or other sensory representation. Mental images seem to be common among individuals with clinical conditions such as eating disorders in which body dissatisfaction is a usual characteristic (Dugue et al., 2016; Somerville et al., 2007), and mental imagery is related to body dissatisfaction among students (Darling et al., 2015). Consequently, exploring the occurrence of mental images among individuals with body dissatisfaction is warranted.

A few studies have investigated the use of imagery in eating disorders or disordered eating (Cooper et al., 2007; Dugue et al., 2019; Ohanian, 2002; Pennesi \& Wade, 2018; Zhou et al., 2020). However, there is paucity of research on ImRS in relation to body image dissatisfaction. The mechanisms through which imagery rescripting as a stand-alone intervention might affect body image and associated emotions, and the extent to which this might lead to body image deserve further investigation.

The ImRS intervention administered in the current study was based on the steps outlined in Hackmann et al. (2011), and Holmes et al. (2019). Participants were instructed to bring to mind the most intrusive and distressing mental body-related image, and to describe the accompanying characteristics of the negative image, as well as triggers and other personal appraisals associated with the image. Then, they were guided to find an 'antidote' to the previously identified negative emotion and meaning, and to implicitly embody a more reflective and compassionate stance. The latter was done through questions such as what they would say to a child that was feeling this way about herself. Next, they were instructed to imagine an image in which they are experiencing the antidote emotion and meaning, and to describe the characteristics of this antidote image. Subsequently, they were asked to revisit the original negative image for a few seconds and notice the emotions, and then to insert the new antidote image in mind and reflect upon its meaning. In the next stage the participant was guided to alter some aspects such as the believability of the original negative 
image in a way that would be impossible in reality (e.g., by making it look funny).

\section{Aims}

Our first aim was to investigate the occurrence of bodyrelated mental images and their associated frequency, positive or negative valence, type, intensity, duration, perspective (observer or field), vividness, emotional consequences, and cognitive content in a convenience sample of female university students with various levels of body dissatisfaction (Study 1). Our second aim was to study the feasibility and initial effects (Study 2) of a single session of imagery rescripting (ImRS) based on a specific intervention protocol targeting negative body-related mental images compared to a credible placebo condition (Expressive writing about body related thoughts and emotions: EW). Our third aim was to investigate the efficacy of ImRS in a larger randomized controlled trial (Study 3) by comparing ImRS to not only EW, but also a wait-list control conditions.

We hypothesized that participants would report a variety of body-related mental images, with positive associations between the frequency of negative body-related mental images and body dissatisfaction (study 1). In addition, a single session of ImRS would have an impact on the properties of the most recurring body-related negative image immediately after the intervention, and the intervention would lead to changes in body dissatisfaction and eating disorders psychopathology at 1-month follow-up (study 2: pilot trial). Furthermore, it was hypothesized that findings of the pilot trial would be replicated and extended in a randomized controlled trial and we would also observe a significant impact of imagery rescripting on body dissatisfaction at the shortterm follow-up (one week) and possibly at the longer-term follow-up (one month).

\section{Methods}

Study 1 was a small cross-sectional study to investigate the occurrence of body-related mental images and the relationship of such images with body shape dissatisfaction, using both quantitative and qualitative analyses. Study 2 was a pilot randomized controlled trial (RCT) to investigate the efficacy of imagery rescripting compared to an active placebo (Expressive writing). Study 3 was a larger RCT comparing ImRS, EW, and a waitlist control condition.

\section{Study 1}

\section{Participants, Instruments and Procedure}

Given the higher prevalence of body dissatisfaction among females compared to males, only females were included in this pilot study. Participants $(\mathrm{n}=31)$ were females, and mainly university students $(84.7 \%)$, with a mean age of 27.8 years $(\mathrm{SD}=9.7)$. They were asked to respond to a brief self-report questionnaire (paper and pencil) on the occurrence, frequency, and characteristics of body-related mental images (Online Appendix 1), the 8-item version of the Body Shape Questionnaire (BSQ) (Welch et al., 2012), and demographic questions regarding gender, age, education, and occupation, as well as current mood (using a visual analog scale ranging from not at all depressed to very depressed). Flyers were set up at designated areas within the campus and interested individuals contacted the research team for participation. Participants received information about the study, responded to the questionnaire and received a cinema ticket for their participation. No identifying information was collected in study 1 , as planned. The study was approved by the Regional Ethical Committee in (Masked information for anonymous review).

\section{Qualitative and Statistical Analysis}

The nature of the described body-related mental images was investigated using thematic analysis. Basic descriptive analyses, and correlations (Pearson or Spearmen) were used to investigate the relationship between body dissatisfaction and occurrence, frequency, or characteristics of mental images (e.g., vividness, intensity, duration, use of first-or third-person perspective, the emotion elicited by the image, and negative or positive emotional load).

\section{Results and Discussion}

All the participants reported at least one body-related mental image. The first reading of the described images by the first author and another independent researcher created an impression of a variety of scenarios, which despite significant variations were readily divided into a positive or a negative theme. This was corroborated in further readings of the text, and inclusion of another independent researcher, with complete agreement between all three.

The positive emotional theme included the following scenarios: future nice haircuts and hairstyles, pleasant sessions of exercise workout (past and present) focusing on muscles and feeling strong, being mindful of the body (e.g., while walking and sensing the wind), being on the move (e.g., on the beach) and sensing freedom and strength, future weight loss, and to put on favorite jeans and feeling how well they fit.

The negative emotional theme included looking critically at one's body in the mirror, past experiences of pregnancy, current negative consequence of past pregnancies on the body, misfit of different outfits, being big, heavy and clumsy, 
bad skin, pimples, big belly, aging body, being boney with many scars, being too fat, looking bigger than one actually is, and a dysfunctional body.

The relationship between body dissatisfaction and frequency of mental imagery in general and body-related imagery, as well as characteristics of body-related imagery are shown in Table 1. Frequency of body-related mental imagery and negative valence of such images were significantly related to body dissatisfaction (Table 1).

Our results indicate the occurrence of both negative and positive mental images. As this was a small sample, the focus was not merely on the significance testing, but also the magnitude of the associations (correlations). As expected, the frequency of mental imagery in general (i.e., how often they have various mental images in mind) was not related to body dissatisfaction. However, we found a significant correlation between the frequency of body-related imagery and body dissatisfaction, as well as between negative valence of images and body dissatisfaction. Interestingly, we found neither a significant, nor a sizeable negative correlation between positive valence of body-related mental images and body dissatisfaction. Measuring the perspective from which the mental image was seen (i.e., first or third perspective) as well as the time frame of the mental image (past, present or future) on a visual analog scale might be a non-optimal choice, or result in low sensitivity of the measurement. This might in turn partly contribute to the non-significant correlations with body dissatisfaction. However, these facets of body-related mental image might also be unrelated to body

Table 1 The association between body dissatisfaction measured by the Body shape questionnaire and frequency of Mental Imagery (in General and Body-Related), and Characteristics of the Body-Related Mental Imagery $(\mathrm{N}=31)$ in Study 1

\begin{tabular}{ll}
\hline & Statistical analysis \\
\hline Frequency of mental images in general $^{\mathrm{a}}$ & $r=0.31, p=0.09$ \\
Frequency of body-related image $^{\mathrm{a}}$ & $r=0.44, p=0.02$ \\
Vividness of the images $^{\mathrm{b}}$ & $r=0.21, p=0.26$ \\
Negative valence of the image $^{\mathrm{c}}$ & $r=0.37, p=0.04$ \\
Positive valence of the image $^{\mathrm{d}}$ & $r=-0.22, p=0.26$ \\
Third-, or first perspective $^{\mathrm{e}}$ & $r=0.20, p=0.29$ \\
Past, now or future nature of the image $^{\mathrm{f}}$ & $r_{s}=0.16, p=0.39$ \\
\hline
\end{tabular}

Pearson's product moment correlation was used in all analyses with the exception of last one (Spearman)

Variables related to imagery were rated on a $133 \mathrm{~mm}$ long Visual Analog Scale (VAS)

${ }^{\text {a }}$ VAS from Very rarely to Very often

${ }^{\mathrm{b}}$ VAS from Very vague to Very clear

${ }^{\mathrm{c}}$ VAS from Not at all negatively charged to Very negatively charged

${ }^{\mathrm{d}}$ VAS from Not at all positively charged to Very positively charged

${ }^{\text {e}}$ VAS from Completely outside to Completely inside

${ }^{\mathrm{f}}$ VAS from Far in the past, Present in the middle, to Far in the future dissatisfaction even if the measurements would have been optimal and sensitive.

Taken together, results suggest that targeting negative body-related mental images might be a plausible choice in an intervention based on imagery rescripting.

\section{Study 2 (Pilot trial)}

\section{Design}

This was a randomized controlled pilot trial with two conditions: Imagery Rescripting (ImRS) and Expressive Writing $(\mathrm{EW})$, with repeated measures across time (pre-, and postintervention assessment, as well as 1 and 4 week later). EW has been previously used as credible intervention or placebo conditions in studies on body image and eating disorders (e.g., Lafont \& Oberle, 2014; Stice et al., 2006). The primary outcome was the level of body dissatisfaction based on the Body Shape Questionnaire (BSQ: Welch et al., 2012). Potential processes through which any change due to ImRS might occur were believability, vividness, controllability, negative affect, and distress related to the most recurrent body-related mental image. Secondary outcomes were between groups differences with regard to eating disorders symptoms, quality of life, symptoms of anxiety and depression, and internalization of the thin ideal as described below.

\section{Participants, Instruments and Procedure}

Participants were in geographical proximity to the university and recruited through advertisements about the study using social media (Instagram and Facebook). Clicking the advertisements opened the web page of the study where those interested could read about the study and register their interest for participation.

Eligible participants (females, 18-45 years old, with a subjective sense of body dissatisfaction) were asked to provide written and informed consent (Regional Ethics Committee in "Masked information for anonymous review") and to respond to a set of questionnaires through a secure server. To avoid stigmatization, and for generalization of outcome, only a subjective sense of body dissatisfaction was the primary requirement for participation instead of a specific level of body dissatisfaction.

The questionnaires consisted of the brief version of the BSQ (BSQ: Welch et al., 2012) to measure body dissatisfaction, the eating disorder examination questionnaire (EDE-Q: Fairburn \& Beglin, 1994; Welch et al., 2011) to measure specific psychopathology of ED at baseline and the 1-month follow-up, the Brunnsviken brief quality of life scale (BBQ: Lindner et al., 2016) to assess quality of life within several main areas of life, the hospital anxiety and depression scale (HADS: Zigmond \& Snaith, 1983), the ideal body stereotype 
scale-revised (IBSS: Stice et al., 2006) to examine the thinideal internalization, and the credibility/expectancy questionnaire (CEQ: Devilly \& Borkovec, 2000). The internal consistency of each instrument (Cronbach's alpha) was as follows: The BSQ (0.84), the EDE-Q total score (0.87), the BBQ (0.76), the HADS-depression (0.78), HADS-anxiety (0.70), the IBSS (0.72), and CEQ (0.79).

Those who reported current suicidality, high levels of depressive symptomatology, regular objective binge eating, or regular purging were excluded from participation and offered information to access clinical services. Eligible participants were asked to provide their full contact information before they were randomized and received their invitation to attend a single intervention session. Of the 179 who responded to the advertisement, 60 were included in the study and randomly assigned to $\operatorname{ImRS}\left(\mathrm{N}=30, \mathrm{M}_{\mathrm{age}}=33.1\right.$, $\mathrm{SD}=7.8)$ or $\mathrm{EW}\left(\mathrm{N}=30, \mathrm{M}_{\mathrm{age}}=35.7, \mathrm{SD}=8.6\right)$ using a randomization list obtained from www.randomizer.org. To prevent deliberate or unconscious interference with the randomization process, the research assistants and the study coordinator had no access to the randomization list. Once a participant met all the criteria for inclusion, her study code was immediately sent to the first author who declared the condition to which the participant should be assigned according to the randomization list. The flow of participants, reasons for exclusion and numbers at follow-up are illustrated in Fig. 1. Of those randomized, 17 (28.3\%) had completed primary or high school, five $(8.3 \%)$ had completed vocational training, and $38(63.3 \%)$ had completed at least 1 year of college or university studies.

Participants were then asked to attend a single session of ImRS or EW at the university. Those in the ImRS were asked to describe their most recurrent body-related negative mental image and to rate its properties (frequency, believability, vividness, controllability, as well as related negative affect and distress). The frequency was measured using the following choices (Several times a day, Once a day, A couple of time/week, Several times a month) before the rescripting. The other properties of the mental image (i.e., vividness, etc.) were measured using visual analog scales from Not at all to Very Much) before and after rescripting, and at followups. The specific rating sheets for assessments at the start and end of the session were developed by the research group based on measurements used in previous imagery studies (Ritter \& Stangier, 2016; Steil et al., 2011; Wild et al., 2008), and established procedures (Holmes et al., 2019). The assessment sheets can be obtained by contacting the corresponding author.

Seven daily rating sheets were also given to participants to obtain daily responses on the frequency (number of times the image occurred and imagery techniques were practiced), the meaning, and properties (believability, vividness, controllability, negative affect and distress) of their negative mental body-related mental images throughout the seven consecutive days following the intervention. They were instructed to use the techniques practiced during the session for the next seven consecutive days whenever a negative mental image of the body appeared or when thoughts about their body emerged.

Those in the EW were instructed to write about thoughts and emotions related to negative body image for the same duration as the ImRS session. As a homework assignment, they were asked to complete $20 \mathrm{~min}$ of writing for the next seven consecutive days. Participants were told to keep the writings to themselves, but were asked to record the date and time of writing each day on a simple recording sheet. At the end of the week, participants were asked to post these respective sheets using a pre-paid envelop, and then respond to the same set of questionnaires that were completed at baseline through a designated web portal (upon receiving a link) one month after the face-to-face meeting.

\section{Intervention}

The ImRS intervention administered was similar to the steps outlined in Hackmann et al. (2011), and the imagery rescripting chapter in Holmes et al. (2019), and adapted where appropriate in accordance with the purpose of the present study. The therapist first provided a definition of mental imagery. In Stage 1, participants were instructed to close their eyes and bring to mind the most intrusive and distressing mental body-related image. Participants were then questioned on the accompanying characteristics of the negative image, including emotions and meanings described in the first person present tense (e.g., How does the image make you feel emotionally? What is the meaning of this negative image?). Afterwards, participants completed the pre-ImRS rating sheet. In Stage 2, participants were asked to describe triggers and other personal appraisals associated with the image (What usually triggers this image to pop up? What would others think of you if the image was true?). The researcher then verbally guided the participant in finding the antidote to the previously identified negative emotion and meaning (e.g., When you have this image in your mind and it makes you feel disgusting, and thinking that you are not good enough, what would be the exact opposite of this emotion and meaning?). Questions that enabled participants to implicitly embody a more reflective and compassionate stance were also asked (e.g., What would you say to a child or someone you cared about if they were feeling this way about themselves?). In Stage 3 , the researcher instructed the participant to imagine an image in which they are experiencing the antidote emotion and meaning. Participants were asked to describe the characteristics of this antidote image (e.g., what is happening in the image right now? How does that make you 


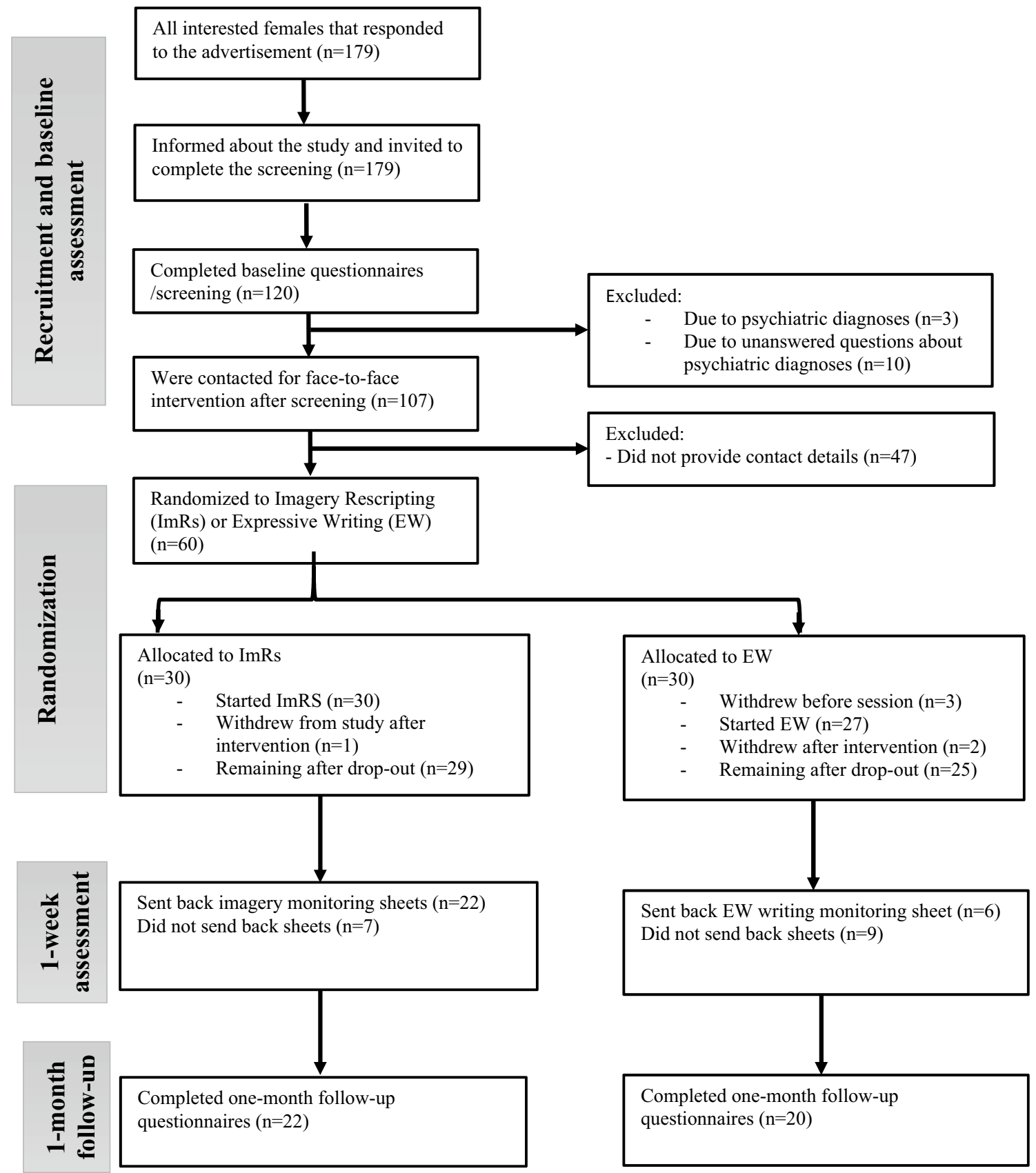

Fig. 1 The flow of Participants through the Pilot Trial and Randomization to Imagery Rescripting (ImRS) or Expressive Writing (EW) in Study 2

feel?). Subsequently, they were told to revisit the original negative image, hold it in mind for a few seconds and feel how the image made them feel. They were then asked to insert the new antidote image in mind whilst the affect was still strong and reflect upon its meaning. In Stage 4, the researcher then verbally guided the participant through several exercises where aspects of the original negative image were altered in some way. The goal was to change the image in a way that would be impossible in reality as a means to facilitate the recognition that negative images are mental representations and not reality in itself. This was executed in the current study by rescripting the believability of the existing negative image using different meta-cognitive techniques. For example, introducing or manipulating aspects of the original image to make it look funny, or putting the image in a bottle and throwing it away into the ocean. After this, participants completed the post-intervention rating sheet and provided ratings on 
how they felt after modifying their images. The session lasted approximately $60 \mathrm{~min}$.

Several psychology undergraduate students (all completing their last year of the 5-year MS in clinical psychology) were trained and supervised to deliver the interventions.

For the EW condition, the researcher provided verbal instructions that encouraged participants to "let go" and explore their deepest thoughts and emotions linked to their body. Participants were asked to write about their most troubling and upsetting experiences, thoughts and memories. They were informed that no one would read their writing and were told to write continuously for the duration of $30 \mathrm{~min}$. The writing was completed quietly in a private and comfortable room. Participants were debriefed after the session, and were instructed to take their writing home. Confidentiality and anonymity were assured in order to enhance compliance to the writing instructions given.

\section{Statistical Analyses}

All the analyses were performed in SPSS version 25 (IBM Corp, 2017). Analysis of variance was used to explore time* group interaction with regard to primary and secondary outcome. Within group effects of process measure in the ImRS condition were investigated using repeated measures t-test. Effect sizes (Cohen's d or partial eta squared) are presented along with the test of statistical significance. Given the mechanistic nature of the study and in line with hypotheses, only completers analyses were of interest. Completers were defined as those who did the task and provided data, see Fig. 1.

\section{Results and Discussion}

No statistically or clinically meaningful differences emerged between the groups on demographic or outcome measures at baseline. Participants perceived both interventions as credible and meaningful with no statistically significant differences in credibility $(t(58)=0.22, p=0.83)$ or expectancy $(t(58)=-1.10, p=0.28)$.

Of the 60 randomized participants, 42 completed the trial and provided data, thus meeting the criteria for completer analysis (Fig. 1). Consequently, the analyses are conducted on this sample $\mathrm{N}=42$. The drop-out group $(\mathrm{N}=18$ : 9 early and 9 later drop-outs) was not significantly different from completers on any baseline measures.

Examples of participants' most intrusive and distressing body-related mental images are presented in Online Appendix 2. They are indicative of a large variation in regards to content and related emotions.
The mean and standard deviation of primary and secondary outcomes before the intervention and one month later are presented in Table 2.

The ANOVA showed a significant effect of time (i.e., baseline to 1-month follow-up) on the BSQ, $F(1,38)=8.44$, $p=0.006, \eta_{p}{ }^{2}=0.18$, and the EDE-Q total score, $F(1$, $35)=8.76, p=0.005, \eta_{p}{ }^{2}=0.20$. However, there were no group $\left(F(1,38)=1.05, p=0.31, \eta_{p}{ }^{2}=0.03\right)$, or group*time interaction effect for the BSQ $(F(1,38)=3.01, p=0.09$, $\left.\eta_{p}{ }^{2}=0.07\right)$. None of the conditions were superior to the other one. The duration of ImRS session (60 $\mathrm{min}$ ) was twice as long as the EW session. This is a confounding variable that might have been even more problematic if the ImRS showed superiority in outcome. Including the session time as a covariate did not change the results.

As the hypothesized superiority of the ImRS compared to the EW in relation to the main and secondary outcome was not supported, it was essential to see whether the suggested mechanisms proposed in ImRS were manipulated as intended. The within-session changes in properties of participant's mental images are presented in Table 3.

As shown in Table 3, all the properties of the negative image changed significantly from baseline to post-intervention with moderate or large effect sizes. Visual inspection of the daily report of the properties of the image during the seven days post-ImRS (Table 4) seems to indicate a favorable change across the week, with decreased frequency, believability, vividness, negative affect and distress, as well as increased controllability of the image. Participants in the EW condition spent 16.00 min daily $(\mathrm{SD}=7.76)$ on doing the EW homework assignment.

Non-parametric analysis of data across the week for frequency of the image revealed a non-significant trend

Table 2 Primary and secondary outcome from baseline to one-month follow-up in the ImRS condition $(\mathrm{N}=22)$, and $\mathrm{EW}$ condition $(\mathrm{N}=20)$ in Study 2

\begin{tabular}{llllll}
\hline & \multicolumn{3}{l}{ Pre-Intervention: M (SD) } & \multicolumn{2}{l}{$\begin{array}{l}\text { 1-month follow-up: M } \\
\text { (SD) }\end{array}$} \\
\cline { 2 - 3 } \cline { 5 - 6 } & ImRS & EW & & ImRS & EW \\
\hline BSQ & $22.19(6.09)$ & $21.89(5.77)$ & $21.14(7.36)$ & $17.74(6.08)$ \\
EDE-Q total & $2.61(1.18)$ & $2.96(1.10)$ & $2.38(1.32)$ & $2.27(1.09)$ \\
BBQ & $35.81(5.82)$ & $35.00(6.32)$ & $36.81(4.34)$ & $35.26(7.23)$ \\
$\begin{array}{l}\text { HADS- } \\
\quad \text { Anxiety }\end{array}$ & $10.00(2.00)$ & $10.50(2.39)$ & $9.78(2.84)$ & $9.94(3.53)$ \\
$\begin{array}{l}\text { HADS- } \\
\quad \text { Depression }\end{array}$ & $8.94(1.80)$ & $9.06(2.01)$ & $9.67(1.85)$ & $8.5(1.41)$ \\
IBSS & $21.38(2.94)$ & $21.16(3.86)$ & $22.24(2.76)$ & $20.84(3.24)$ \\
\hline
\end{tabular}

$B S Q$ Body Shape Questionnaire, EDE- $Q$ total Eating Disorder Examination Questionnaire global score, $B B Q$ Brunnsviken Brief Quality of Life Scale, HADS Hospital Anxiety and Depression Scale respectively, and IBSS Ideal Body Stereotype Scale, respectively 
Table 3 Mean (M) and Standard Deviation (SD) of the Properties of Negative Body-Related Mental Images from before to after ImRS (N=29) along Within-Group $t$-test and Related Effect Size (Cohen's d) within the ImRS Group only, in Study 2

\begin{tabular}{llll}
\hline & Before ImRS $M(S D)$ & After ImRS $M(S D)$ & $t$-test, $p$, and effect size $($ Cohen's $d)$ \\
\hline Believability & $11.18(3.63)$ & $9.23(4.21)$ & $t(28)=2.79, p=0.009, d=0.52$ \\
Vividness & $12.48(3.30)$ & $8.07(4.23)$ & $t(28)=6.39, p<0.001, d=1.22$ \\
Controllability & $7.36(4.24)$ & $9.14(3.74)$ & $t(28)=-2.17, p=0.039, d=0.41$ \\
Negative affect & $13.09(2.49)$ & $7.60(4.19)$ & $t(28)=6.71, p<0.001, d=1.31$ \\
Distress & $12.53(2.32)$ & $7.15(3.88)$ & $t(28)=7.43, p<0.001, d=1.46$ \\
\hline
\end{tabular}

Table 4 Means and standard deviations of number of times the participants practiced imagery on each particular day (Rescript), and the properties of the image (i.e., Frequency, Believability,etc.) During the Week following the ImRS Session in the ImRS Condition Only $(\mathrm{N}=21)$ in Study 2

\begin{tabular}{llllllll}
\hline Day & Rescript & Frequency & Believability $^{\mathrm{a}}$ & Vividness $^{1}$ & Control $^{1}$ & Affect $^{1}$ & Distress $^{\mathrm{a}}$ \\
\hline 1 & $1.43(1.08)$ & $2.05(1.16)$ & $10.34(4.67)$ & $10.29(4.92)$ & $8.21(5.33)$ & $9.82(4.53)$ & $7.92(5.35)$ \\
2 & $1.48(.98)$ & $2.14(1.15)$ & $10.75(4.65)$ & $10.60(4.22)$ & $7.19(4.78)$ & $9.70(3.83)$ & $7.52(4.98)$ \\
3 & $1.81(1.17)$ & $2.38(1.32)$ & $9.99(4.62)$ & $9.61(4.56)$ & $7.81(5.00)$ & $9.06(4.96)$ & $7.52(4.85)$ \\
4 & $1.48(1.36)$ & $1.76(1.37)$ & $8.09(5.52)$ & $7.03(5.61)$ & $9.41(5.21)$ & $7.43(5.62)$ & $5.99(5.73)$ \\
5 & $1.38(1.02)$ & $1.95(1.12)$ & $9.16(4.94)$ & $8.53(4.95)$ & $8.52(5.46)$ & $8.92(5.10)$ & $7.29(4.97)$ \\
6 & $1.55(1.10)$ & $1.95(1.28)$ & $9.66(5.26)$ & $9.10(5.55)$ & $8.79(5.51)$ & $8.30(4.99)$ & $6.58(5.04)$ \\
7 & $1.29(1.10)$ & $1.62(1.36)$ & $7.59(5.47)$ & $6.49(5.06)$ & $9.87(5.48)$ & $6.16(4.80)$ & $4.75(4.45)$ \\
\hline
\end{tabular}

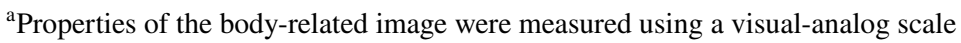

towards a reduction $(p=0.07)$ in Frequency of the image, a significant reduction in the Vividness of the image (Friedman's ANOVA by ranks $=18.81, d f=6, N=21, p=0.004$ ), a non-significant tendency towards an increase $(p=0.07)$ in Control of the image, and significant reductions in the negative Affect (Friedman's ANOVA by ranks $=14.03, d f=6$, $N=21, p=0.03$ ) and Distress (Friedman's ANOVA by ranks $=15.49, d f=6, N=21, p=0.02$ ) related to the image.

Data from 1-month follow-up of the BSQ showed an overall time effect for both conditions (ImRS and EW), but no significant interaction. As the BSQ was not measured at 1-week follow-up, it remained unclear whether the shortterm changes in the properties of the image in the ImRS could have influenced body dissatisfaction one week later. The changes in the properties of the image in this pilot trial were promising enough to further investigate whether they could accompany changes in body dissatisfaction after one week of daily use of ImRS, and if so, whether they could mediate the change in body dissatisfaction. In addition, to investigate the reactivity of the measures, a wait-list control condition was also included in the next randomized controlled trial.

\section{Study 3 (Randomized Controlled Trial: RCT)}

\section{Participants, Instruments and Procedure}

One hundred and thirteen women (18-45 years old) with a subjective sense of body dissatisfaction were recruited through the same procedures as in the pilot trial and randomized into ImRS $\left(N=40, M_{\mathrm{age}}=33.8, S D=7.3\right)$, EW $\left(N=42, M_{\text {age }}=32.5, S D=8.1\right)$, or a waitlist control condition (WL: $N=31, M_{\text {age }}=31.9, S D=7.9$ ). Participants provide their written and informed consent (Masked information for anonymous review). Given a single session of intervention, a change on body dissatisfaction equal to an effect size between small and medium $(f=0.17)$ was deemed meaningful. Thus, the power analysis of interaction effect with $f=0.17$, for 3 conditions and 2 repeated assessments, suggested a total sample size of 87 for a power of at least 0.80 with $\mathrm{p}<0.05$. To consider the risk of drop-out, based on the observation in study 2 (30\% drop-out), the sample was expanded to 113 . Process measure were obtained only at baseline and after the end of the intervention. The outcome variables were obtained at baseline and at 1-month followup with the exception of the BSQ that was also measured at 1-week follow-up. The total number of those who declared interest to participate and reasons for exclusion are presented in Fig. 2.

The same instruments and similar procedures from the pilot trial were employed in the RCT. However, those in the EW were also asked to describe their most recurrent body-related mental image and its associated properties (e.g., believability, vividness, etc.) both before and after the 40 min session (i.e., Expressive writing) during which participants wrote about their most inner thoughts and emotions related to their body. In addition, a repeated measure of BSQ was obtained at the 1-week follow-up to investigate the possible short-term effect of the interventions. In this RCT, the outcome and the change in 


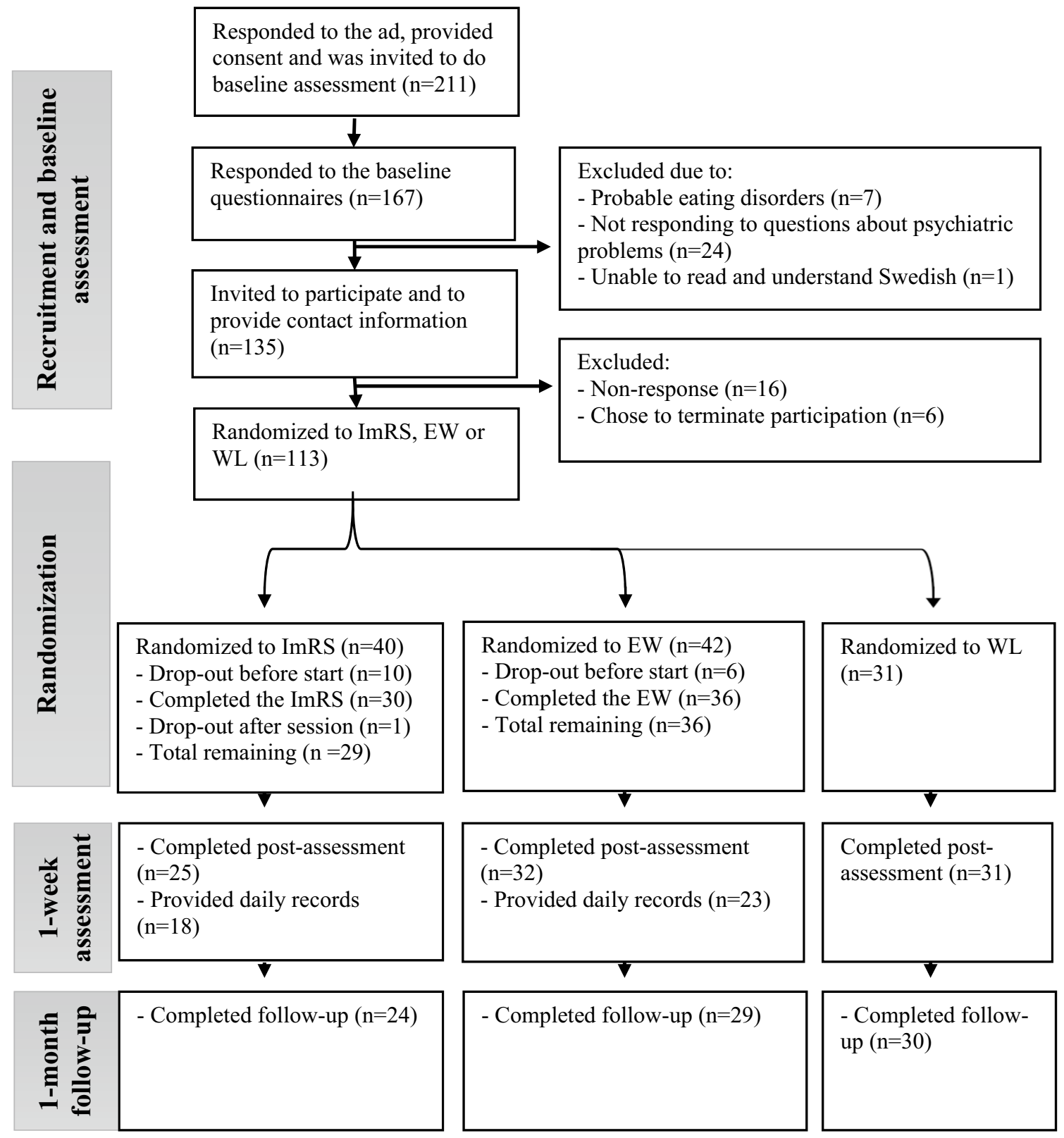

Fig. 2 The Flow Chart Illustrating the Recruitment of Participants, and Randomization to Imagery Rescripting (ImRS), Expressive Writing (EW) or Wait-List (WL) in Study 3

properties of the image were investigated using Generalized linear mixed models (GLMM) to model the data more properly (nesting the observations across time into the participants, and including random intercept) and make use of the flexibility of the GLMM (choosing the distribution and link based on the actual distribution of the outcome variable), and the inherent intention to treat properties of the mixed models.

\section{Results and Discussion}

At baseline, there were no significant differences between the conditions in terms of demographics, the outcome variables, the ratings of credibility or expectancy (ImRS vs EW only), use of social media, or mental imagery in general (thinking through images). 
Of the 40 participants randomized to ImRS and the 42 participants randomized to EW, 29 (72.5\%), and 36 (86\%) completed the intervention, respectively (Fig. 2).

The drop-outs, both in total or within each condition, were not significantly different from completers on any demographics or outcomes at baseline assessment. Changes across the session (before and after ImRS) are described in Table 5.

We found no significant interaction between condition and time for believability or the distress caused by the image, but a significant interaction for vividness, controllability and negative affect caused by the image (Table 5). The same set of questions (believability, vividness, controllability, etc.) were also asked in relation to body-related thoughts (compared to image) in both conditions before and after each intervention. While both groups reported significant improvements with small effects, no significant interactions were found.

After the intervention session, participants in the ImRS were asked to use the ImRS strategies on a daily basis, and those in the EW were asked to write about any thoughts or emotions related to their body for a duration of 30-40 $\mathrm{min}$ per day. A total of 18 out of 25 participants who completed the post-assessment in the ImRS, and 23 out of 32 in the EW completed their assignments. The participants reported spending $19.34(\mathrm{SD}=7.57)$ minutes each day on EW. Subsequently, all participants were asked to respond to the BSQ one week later, and to contribute to the 1-month followup during which several self-report questionnaire were administered.

At 1-week follow-up after baseline, there was a main effect of time for body dissatisfaction $(F(1,193)=24.67$, $p<0.001$, Cohen's $d=1.01$ ). Body dissatisfaction decreased in all three conditions. There was neither a significant main effect of condition $(F(2,193)=0.93, p=0.40$, Cohen's $d=0.20$ ), nor an interaction between time and condition $(F(2,193)=2.46, p=0.088$ Cohen's $d=0.82)$.

The descriptive statistics for baseline and 1-month follow-up are presented in Table 6. At 1-month follow-up, for body dissatisfaction, there was not any significant effect of condition $(F(2,176)=1.63, p=0.198$, Cohen's $d=0.27)$, but a significant time effect $(F(1,176)=65.96, p<0.0001$, Cohen's $d=1.73$ ), and a significant time by condition interaction $(F(2,176)=4.33, p=0.015$, Cohen's $d=0.44)$. However, none of the Sidak post-hoc pairwise comparisons were significant. For the eating disorders symptoms based on the EDE-Q, there was also a significant time effect ( $F(1$, $176)=31.47, p<0.0001$, Cohen's $d=1.20$ ), but not significant effect of condition or interaction between time and condition. Based on the EDE-Q global scores and Swedish

Table 5 Mean (M) and standard error (SE) of the properties of negative images, in Study 3, from before to after imagery rescripting (ImRS, $n=29)$ or expressive writing $(E W, n=35)$ along with inter-

action effect of time and condition in the generalized linear mixed model (GLMM), and effect size (Cohen's d) in study 3

\begin{tabular}{lllllll}
\hline & \multicolumn{2}{l}{ Before Intervention: $\mathrm{M}(\mathrm{SE})$} & & \multicolumn{2}{l}{ After intervention: M (SE) } & Interaction effect in GLMM \\
\cline { 2 - 3 } & ImRS & EW & & ImRS & EW & \\
\hline Believability & $11.33(0.66)$ & $10.59(0.60)$ & & $7.27(0.66)$ & $8.09(0.60)$ & $F(1,128)=3.17, p=0.077, d=0.45)$ \\
Vividness & $12.28(0.58)$ & $11.22(0.53)$ & & $6.67(0.58)$ & $8.39(0.53)$ & $F(1,128)=8.09, p=0.005, d=0.71)$ \\
Controllability & $6.01(0.70)$ & $8.17(0.64)$ & & $11.20(0.70)$ & $10.56(0.64)$ & $F(1,128)=6.21, p=0.014, d=0.62)$ \\
Negative affect & $14.33(0.57)$ & $12.19(0.52)$ & & $8.53(0.57)$ & $9.29(.052)$ & $F(1,128)=9.79, p=0.002, d=0.78)$ \\
Distress & $11.85(0.56)$ & $10.93(0.51)$ & & $6.94(0.56)$ & $7.63(0.51)$ & $F(1,128)=2.76, p=0.099, d=0.42)$ \\
\hline
\end{tabular}

Table 6 Mean and standard error of the primary (BSQ) and Secondary Outcomes at Baseline and 1-Month Follow-Up in the imagery rescripting (ImRS), Expressive writing (EW), and the wait-list Conditions (WL) in Study 3

\begin{tabular}{llllllll}
\hline & \multicolumn{3}{l}{ Baseline assessment } & & & \multicolumn{2}{l}{ 1-month follow-up } \\
\cline { 2 - 3 } & ImRS & EW & WL & & ImRS & EW & WL \\
\hline BSQ & $30.70(0.94)$ & $30.21(0.92)$ & $30.55(1.07)$ & & $25.05(1.20)$ & $23.54(1.13)$ & $27.99(1.13)$ \\
EDE-Q total & $2.83(0.15)$ & $2.68(0.15)$ & $2.77(0.17)$ & & $2.17(0.19)$ & $1.97(0.18)$ & $2.51(0.18)$ \\
BBQ & $51.43(2.81)$ & $54.24(2.74)$ & $54.32(3.19)$ & & $51.07(3.97)$ & $58.01(3.70)$ & $61.45(3.49)$ \\
HADS-anxiety & $10.90(0.56)$ & $10.17(0.56)$ & $10.20(0.64)$ & & $8.48(0.77)$ & $8.83(0.71)$ & $8.44(0.70)$ \\
HADS-dep & $4.77(0.46)$ & $5.26(0.44)$ & $5.14(0.52)$ & & $4.24(0.63)$ & $4.38(0.58)$ & $4.16(0.55)$ \\
IBSS & $22.63(0.58)$ & $23.24(0.52)$ & $22.71(0.54)$ & & $23.21(0.67)$ & $23.58(0.64)$ & $23.15(0.58)$ \\
\hline
\end{tabular}

$B S Q$ Bod Shape Questionnaire, EDE-Q total Eating Disorders Examination Questionnaire global score, $B B Q$ Brunnsviken Brief Quality of Life Questionnaire, HADS-Anxiety Hospital Anxiety Depression Scale—anxiety score, HADS-D Hospital Anxiety Depression Scale-depressive score, IBSS Ideal Body Stereotype Scale 
norms for the EDE-Q (Welch et al., 2011), about $11 \%$ of the sample in study 3 might have suffered from an eating disorder.

For quality of life, there were neither a significant time, nor any significant effect of condition or interaction. For symptoms of anxiety (HADS-A) and depression (HADS-D), only a significant time effect emerged $(F(1,171)=16.80$, $p<0.0001$, Cohen's $d=0.89$ and $F(1,169)=4.66, p=0.032$, Cohen's $d=0.47$ respectively), but not effect of condition or interaction. For internalization of the thin ideal (IBSS), neither time nor any condition or interaction effects were observed.

\section{General Discussion}

Participants in Study 1 reported at least one body-related mental image, and the frequency of body-related mental imagery and negative valence of such images were significantly related to body dissatisfaction. While positive images were also reported, they showed no significant association with body dissatisfaction. Therefore, targeting negative body-related mental imagery during the ImRS intervention appeared to be a reasonable strategy.

In the pilot trial (Study 2), ImRS resulted in favorable changes on all the properties of the participants' target negative body-related mental image at the end of the session. One week later, daily use of ImRS-techniques resulted in significant changes in the vividness, negative affect and distress related to the mental image. However, changes in the ImRS from baseline to 1-month follow-up on body dissatisfaction were not significantly different compared to changes in the EW condition.

In the efficacy RCT (study 3), changes in the properties of the body-related mental-image were measured in both the ImRS and EW conditions. As expected, all the properties of the image changed favorably to a higher extent in the ImRS than in the EW across the session (e.g., for emotion), but we found no differences between any of the conditions, including the wait list at the one-week or one-month follow-up related to body dissatisfaction. Our findings suggest that a single session of imagery rescripting seems to alleviate the negative properties of the image (i.e., has proximal effects as hypothesized in our mechanistic account), but does not bring about a significant change in body dissatisfaction (i.e., transfer downstream to more distal effects). We might speculate that ImRS of more than one recurring negative bodyrelated image, or focus on boosting positive body-related imagery are needed to make an overall difference on body dissatisfaction.

We note that Willson and colleagues (Willson et al., 2016) found clinically meaningful changes after a single session of an imagery treatment for participants with body dysmorphic disorder in a proof-of-concept study with a multiple baseline single case experimental design. Likewise, Pennesi and Wade (2018) observed significant changes in body image acceptance in the ImRS condition compared to a dissonance-based and a wait-list condition after a brief imagery rescripting intervention and daily practice for five consecutive days. They used a very brief online imagery intervention based on previous work on traumatic events in social phobia (Wild \& Clark, 2011). In this version of the imagery, participants were asked to close their eyes and imagine, and then describe in writing, their earliest memory of an unpleasant body experience from the past from an observer's perspective, and in the present with their compassionate self in the room. Lack of data on the properties of image before and after the intervention in the study by Pennesi and Wade (2018) makes it difficult to understand the potential mechanisms through which the effect on body image acceptance occurred. In contrast, although the changes in the properties of the mental image in the short term, and 1-week follow-up in our study appear promising, they do not seem to affect the perceived body dissatisfaction differentially in the ImRS condition compared to the control conditions. Another next step in future research should be to compare the different ImRS strategies used in our versus the Pennesi et al. study (2018) along with mechanisms that might explain the outcome and compare them both to a credible placebo such as EW. Likewise, the working mechanisms in the EW should also be investigated, as it also showed some benefits. Exploring the most troubling and upsetting emotions and thoughts associated with the body is an exposure that might exert some effects through habituation, comprehensive distancing, or other processes involved in inhibitory learning. Another important factor is the measurement of outcome. Pennesi and Wade (2018) used the Body Image-Acceptance and Action Questionnaire (Sadoz et al., 2013) as the outcome measure, which is a measure of body image flexibility while we use the Body Shape Questionnaire (Welch et al., 2012) that captures phenomenological experience of body dissatisfaction.

Future studies also need to control for actual body checking behavior during the follow-up periods. Finally, as guided imagery has shown promising results in targeting experiential and behavioral avoidance as in the study by Alleva and colleagues (2015), specific targets of experiential and behavioral avoidance in relation to body might also be a viable option for ImRS.

Our findings should be interpreted in light of the strengths and limitations of these included studies. The initial descriptive study needs to be extended by increasing the sample size to further investigate the themes or characteristics to be targeted through the intervention. The therapists in our trials were advanced clinical psychology program students in their final (fifth) year with good clinical skills, but future 
studies may benefit from using more skilled therapists and formal checking of fidelity of the interventions. However, research has shown that advanced students in the psychology program can produce intervention effects that are consistent with the outcomes delivered by skilled psychotherapist (Ost et al., 2012). To increase the sensitivity of the design, future studies may also focus on a subgroup of potential participants with high body dissatisfaction. Future studies can build on the current ones to use pre-registration of study design, and procedure that permit later use of open data in line with developments in reproducibility and open science. We did not perform a semi-structured interview to establish potential diagnosis of eating disorders. Based on the global EDE-Q scores and Swedish norms for the EDE-Q about $11 \%$ of the participants in Study 3 might have suffered from eating disorders. Future studies would benefit from conducting interviews to establish potential diagnosis of eating disorders in order separate eating psychopathology from body dissatisfaction.

To conclude, whilst our studies did not show a significant effect of ImRS on body dissatisfaction, changes in the properties of self-reported mental images during the session and one week post-intervention are promising. Such findings provide justification for future studies to further explore the use of ImRS in addressing this widespread problem and seek to increase much needed preventive approaches available in the area of eating disorders.

Supplementary Information The online version contains supplementary material available at https://doi.org/10.1007/s10608-022-10295-z.

Acknowledgements We would like to acknowledge our former master's students Johanna Nyberg, Hanna Renström, Linnéa Norberg, and Niloofar Safari for all their hard work and dedication. We would also like to thank all the participants for their contribution to research.

Funding Open access funding provided by Karolinska Institute. This research did not receive any specific grant from funding agencies in the public, commercial, or not-for-profit sectors.

Data Availability Data will be made available upon appropriate request. Please contact the corresponding author.

\section{Declarations}

Conflict of Interest AG receives royalties from two books, occasional fees for workshops and supervision with focus on eating disorders, and consultancy fees from the Swedish Agency for Health Technology Assessment and Assessment of Social Services. EW and CZ declare no conflict of interest. EAH reports serving on the board of trustees of the charity MQ: Transforming Mental Health, and receives no remuneration for this role. EAH receives royalties from two books on imagery and occasional fees for workshops and invited addresses; receives occasional consultancy fees from the Swedish Agency for Health Technology Assessment and Assessment of Social Services; and reports current grants from The OAK Foundation (OCAY-18-442), the Lu- pina Foundation, and the Swedish Research Council (2017-00957) for other studies than the current work.

Ethical Approval All the studies were approved by Regional Ethics Committee in Stockholm (Dnr. 2014/1387-31, 2016/2132-32, 2018/289-32). The study was conducted in accordance with relevant guidelines and regulations.

Informed Consent Informed written consent was obtained from all the participants.

Research Involving Animal Rights This article does not contain any studies with animals performed by any of the authors.

Open Access This article is licensed under a Creative Commons Attribution 4.0 International License, which permits use, sharing, adaptation, distribution and reproduction in any medium or format, as long as you give appropriate credit to the original author(s) and the source, provide a link to the Creative Commons licence, and indicate if changes were made. The images or other third party material in this article are included in the article's Creative Commons licence, unless indicated otherwise in a credit line to the material. If material is not included in the article's Creative Commons licence and your intended use is not permitted by statutory regulation or exceeds the permitted use, you will need to obtain permission directly from the copyright holder. To view a copy of this licence, visit http://creativecommons.org/licenses/by/4.0/.

\section{References}

Alleva, J. M., Sheeran, P., Webb, T. L., Martijn, C., \& Miles, E. (2015). A meta-analytic review of stand-alone interventions to improve body image. PLOS ONE, 10, e0139177.

Arntz, A. (2011). Imagery rscripting as a therapeuytic technique: Review of clinical trials, basic studies, and research agenda. Journal of Experimental Psychopathology, 3, 189-208.

Baddeley, A. D., \& Andrade, J. (2000). Working memory and the vividness of imagery. Journal of Experimental Psychology: General, $129,126-145$.

Baddeley, A. D., \& Hitch, G. J. (1974). Working memory. In G. $\mathrm{H}$. Bower (Ed.), The psychology of learning and motivation: Advances in research and theory (Vol. III, pp. 47-90). Academic Press.

Cili, S., \& Stopa, L. (2015). Intrusive mental imagery in psychological disorders: is the self the key to understanding maintenance? Front Psychiatry, 6, 103.

Cooper, M., Todd, G., \& Turner, H. (2007). The effects of using imagery tio modify core emotional beliefs in bulimia nervosa: An Experimental pilot study. Journal of Cognitive Psychotherapy: An International Quarterly, 21, 117-122.

Corp, I. B. M. (2017). IBM SPSS Statistics for Macintosh (Version 25). IBM Corp.

Darling, S., Uytman, C., Allen, R. J., Havelka, J., \& Pearson, D. G. (2015). Body image, visual working memory and visual mental imagery. PeerJ, 3, e775.

Devilly, G. J., \& Borkovec, T. D. (2000). Psychometric properties of the credibility/expectancy questionnaire. Journal of Behavior Therapy and Experimental Psychiatry, 31, 73-86.

Dugue, R., Keller, S., Tuschen-Caffier, B., \& Jacob, G. A. (2016). Exploring the mind's eye: Contents and characteristics of mental images in overweight individuals with binge eating behaviour. Psychiatry Research, 246, 554-560. 
Dugue, R., Renner, F., Austermann, M., Tuschen-Caffier, B., \& Jacob, G. A. (2019). Imagery rescripting in individuals with binge-eating behavior: An experimental proof-of-concept study. International Journal of Eating Disorders, 52, 183-188.

Fairburn, C. G., \& Beglin, S. J. (1994). Assessment of eating disorders: Interview or self-report questionnaire? International Journal of Eating Disorders, 16, 363-370.

Fallon, E. A., Harris, B. S., \& Johnson, P. (2014). Prevalence of body dissatisfaction among a United States adult sample. Eating Behaviors, 15, 151-158.

Fiske, L., Fallon, E. A., Blissmer, B., \& Redding, C. A. (2014). Prevalence of body dissatisfaction among United States adults: Review and recommendations for future research. Eating Behaviors, 15, $357-365$.

Ghaderi, A. (2003). Structural modeling analysis of prospective risk factors for eating disorder. Eating Behaviors, 3, 387-396.

Griffiths, S., Murray, S. B., Bentley, C., Gratwick-Sarll, K., Harrison, C., \& Mond, J. M. (2017). Sex differences in quality of life impairment associated with body dissatisfaction in adolescents. Journal of Adolescent Health, 61, 77-82.

Hackmann, A., Bennett-Levy, J., \& Holmes, E. A. (2011). Oxford Guide to Imagery in Cognitive Therapy. Oxford University Press.

Holmes, E. A., \& Mathews, A. (2010). Mental imagery in emotion and emotional disorders. Clinical Psychology Review, 30, 349-362.

Holmes, E. A., Mathews, A., Mackintosh, B., \& Dalgleish, T. (2008). The causal effect of mental imagery on emotion assessed using picture-word cues. Emotion, 8, 395-409.

Holmes, E. A., Ghaderi, A., Harmer, C. J., Ramchandani, P. G., Cuijpers, P., Morrison, A. P., \& Craske, M. G. (2018). The Lancet Psychiatry Commission on psychological treatments research in tomorrow's science. Lancet Psychiatry, 5, 237-286.

Holmes, E. A., Hales, S. A., Young, K., \& Di Simplicio, M. (2019). Imagery-based cognitive therapy for bipolar disorder and mood instability. Guilford Publications.

Kearney-Cooke, A., \& Striegel-Moore, R. (1997). The etiology and treatment of body image disturbances. In D. M. Garner \& P. E. Garfinkel (Eds.), Handbook of treatment for eating disorders (pp. 295-306). The Guilford Press.

Lafont, J., \& Oberle, C. D. (2014). Expressive writing effects on body image: Symptomatic versus asymptomatic women. Psychology (savannah, Ga.), 5, 431-440.

Lindner, P., Frykheden, O., Forsstrom, D., Andersson, E., Ljotsson, B., Hedman, E., \& Carlbring, P. (2016). The brunnsviken brief quality of life scale (BBQ): Development and psychometric evaluation. Cognitive Behaviour Therapy, 45, 182-195.

Mond, J., Mitchison, D., Latner, J., Hay, P., Owen, C., \& Rodgers, B. (2013). Quality of life impairment associated with body dissatisfaction in a general population sample of women. BMC Public Health, 13, 920.

Morina, N., Lancee, J., \& Arntz, A. (2017). Imagery rescripting as a clinical intervention for aversive memories: A meta-analysis. Journal of Behavior Therapy and Experimental Psychiatry, 55, $6-15$.

Ohanian, V. (2002). Imagery rescripting within cognitive behavior therapy for bulimia nervosa: An illustrative case report. International Journal of Eating Disorders, 31, 352-357.

Ost, L. G., Karlstedt, A., \& Widen, S. (2012). The effects of cognitive behavior therapy delivered by students in a psychologist training program: An effectiveness study. Behavior Therapy, 43, 160-173.

Paxton, S. J., Neumark-Sztainer, D., Hannan, P. J., \& Eisenberg, M. E. (2006). Body dissatisfaction prospectively predicts depressive mood and low self-esteem in adolescent girls and boys. Journal of Clinical Child and Adolescent Psychology, 35, 539-549.

Pearson, J., Naselaris, T., Holmes, E. A., \& Kosslyn, S. M. (2015). Mental imagery: functional mechanisms and clinical applications. Trends in Cognitive Sciences, 19, 590-602.
Pennesi, J. L., \& Wade, T. D. (2018). Imagery rescripting and cognitive dissonance: A randomized controlled trial of two brief online interventions for women at risk of developing an eating disorder. International Journal of Eating Disorders, 51, 439-448.

Ritter, V., \& Stangier, U. (2016). Seeing in the Mind's eye: Imagery rescripting for patients with body dysmorphic disorder. A single case series. Journal of Behavior Therapy and Experimental Psychiatry, 50, 187-195.

Riva, G. (1998). Modifications of body-image induced by virtual reality. Perceptual and Motor Skills, 86, 163-170.

Riva, G., Bacchetta, M., Cesa, G., Conti, S., \& Molinari, E. (2003). Six-month follow-up of in-patient experiential cognitive therapy for binge eating disorders. Cyberpsychology \& Behavior, 6 , 251-258.

Romano, M., Moscovitch, D. A., Huppert, J. D., Reimer, S. G., \& Moscovitch, M. (2020). The effects of imagery rescripting on memory outcomes in social anxiety disorder. Journal of Anxiety Disorders, 69, 102169.

Sadoz, E. K., Wilson, K. G., \& Mervin, R. M. (2013). Assessment of body image flexibility: The body image-acceptance and action questionnaire. Journal of Contextual Behavioral Science, 2, $39-48$.

Somerville, K., Cooper, M., \& Hackmann, A. (2007). Spontaneous imagery in women with bulimia nervosa: An investigation into content, characteristics and links to childhood memories. Journal of Behavior Therapy and Experimental Psychiatry, 38, 435-446.

Steil, R., Jung, K., \& Stangier, U. (2011). Efficacy of a two-session program of cognitive restructuring and imagery modification to reduce the feeling of being contaminated in adult survivors of childhood sexual abuse: A pilot study. Journal of Behavior Therapy and Experimental Psychiatry, 42, 325-329.

Stice, E., Presnell, K., \& Spangler, D. (2002). Risk factors for binge eating onset in adolescent girls: A 2-year prospective investigation. Health Psychology, 21, 131-138.

Stice, E., Shaw, H., Burton, E., \& Wade, E. (2006). Dissonance and healthy weight eating disorder prevention programs: A randomized efficacy trial. Journal of Consulting and Clinical Psychology, 74, 263-275.

Stice, E., Marti, C. N., Shaw, H., \& Rohde, P. (2019). Meta-analytic review of dissonance-based eating disorder prevention programs: Intervention, participant, and facilitator features that predict larger effects. Clinical Psychology Review, 70, 91-107.

Welch, E., Birgegard, A., Parling, T., \& Ghaderi, A. (2011). Eating disorder examination questionnaire and clinical impairment assessment questionnaire: General population and clinical norms for young adult women in Sweden. Behaviour Research and Therapy, 49, 85-91.

Welch, E., Lagerstrom, M., \& Ghaderi, A. (2012). Body shape questionnaire: Psychometric properties of the short version (BSQ-8C) and norms from the general Swedish population. Body Image, 9, 547-550.

Wheatley, J., Brewin, C. R., Patel, T., Hackmann, A., Wells, A., Fisher, P., \& Myers, S. (2007). I'll believe it when I can see it: Imagery rescripting of intrusive sensory memories in depression. Journal of Behavior Therapy and Experimental Psychiatry, 38, 371-385.

Wild, J., \& Clark, D. M. (2011). Imagery rescripting of early traumatic memories in social phobia. Cognitive and Behavioral Practice, $18,433-443$.

Wild, J., Hackmann, A., \& Clark, D. M. (2008). Rescripting early memories linked to negative images in social phobia: A pilot study. Behavior Therapy, 39, 47-56.

Willson, R., Veale, D., \& Freeston, M. (2016). Imagery rescripting for body dysmorphic disorder: a multiple-baseline single-case experimental design. Behavior Therapy, 47, 248-261.

Zhou, Y., Pennesi, J. L., \& Wade, T. D. (2020). Online imagery rescripting among young women at risk of developing an eating 
disorder: A randomized controlled trial. International Journal of Eating Disorders, 53, 1906-1917.

Zigmond, A. S., \& Snaith, R. P. (1983). The hospital anxiety and depression scale. Acta Psychiatrica Scandinavica, 67, 361-370.
Publisher's Note Springer Nature remains neutral with regard to jurisdictional claims in published maps and institutional affiliations. 